Chapter II

Review Papers

Globular Clusters in the Milky Way 


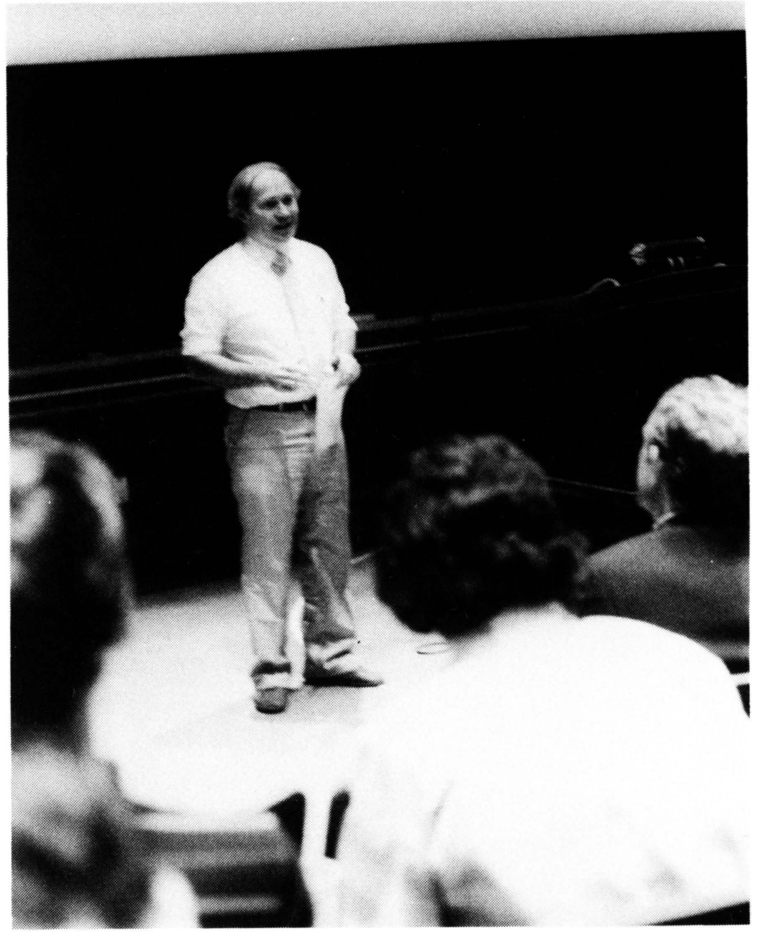

Bob McClure running his session

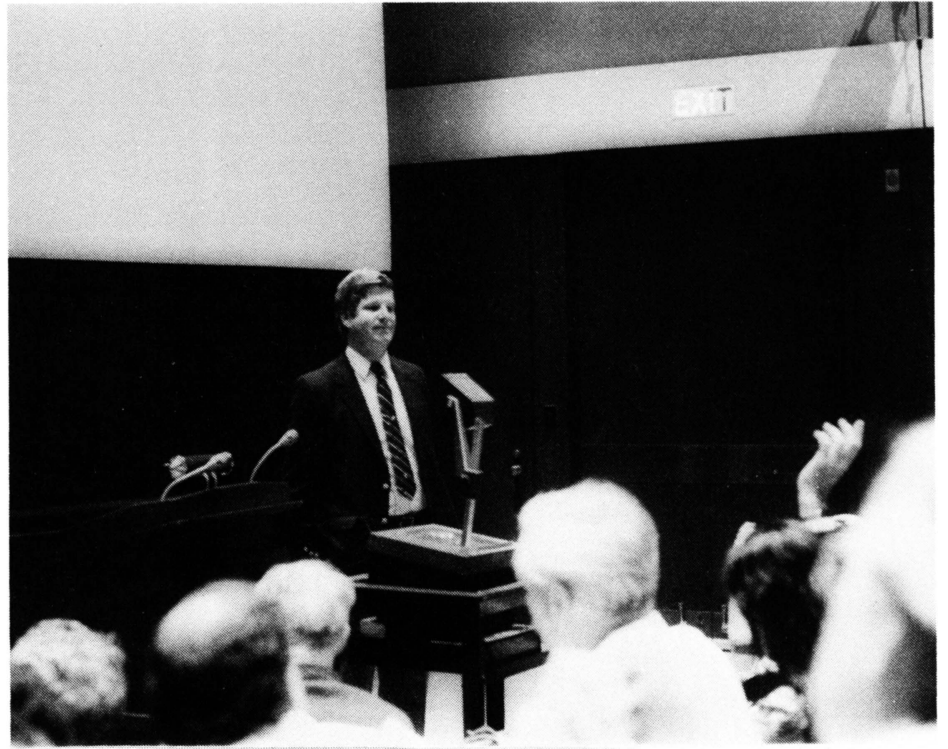

Ron Webbink answering questions after his talk 


\title{
AN OVERVIEW OF THE GLOBULAR CLUSTER SYSTEM OF THE GALAXY
}

\author{
Robert Zinn \\ Yale University Observatory
}

\section{INTRODUCTION}

Harlow Shapley (1918) used the positions of globular clusters in space to determine the dimensions of our Galaxy. His conclusion that the Sun does not lie near the center of the Galaxy is widely recognized as one of the most important astronomical discoveries of this century. Nearly as important, but much less publicized, was his realization that, unlike stars, open clusters, HII regions and planetary nebulae, globular clusters are not concentrated near the plane of the Milky Way. His data showed that the globular clusters are distributed over very large distances from the galactic plane and the galactic center. Ever since this discovery that the Galaxy has a vast halo containing globular clusters, it has been clear that these clusters are key objects for probing the evolution of the Galaxy. Later work, which showed that globular clusters are very old and, on average, very metal poor, underscored their importance. In the spirit of this research, which started with Shapley's, this review discusses the characteristics of the globular cluster system that have the most bearing on the evolution of the Galaxy.

\section{SUBSYSTEMS}

Roughly 150 globular clusters have been identified in the Galaxy, and the majority of them are distributed in a roughly spherical volume around the galactic center. It is very important to ascertain whether all globular clusters belong to this halo population or whether the cluster system contains subsystems with distinct properties that reflect different origins.

Starting with the suggestion by Baade (1958) that the metal-rich globular clusters belong to a disk system, there have been a number of investigations of the correlations between metal abundance, spatial distribution, and kinematics to see whether the clusters divide into distinct groups. It was clear from the first spectroscopic surveys of 
the cluster system (Mayall 1946; Morgan 1956, 1959; Kinman 1959) that the metal-rich clusters are more concentrated towards the galactic center than the metal-poor ones, but until recently the data on the metallicities, distances, and radial velocities of the clusters near the galactic center were inadequate to tell whether the metal-rich clusters are a separate population or simply the metal-rich tail of the halo population. Now there is substantial evidence that the metal-rich clusters constitute a distinct disk system (Zinn 1985), and some of this evidence is reviewed here (see also Zinn 1986).

The distribution of the distances of the globular clusters from the galactic plane shows a sharp discontinuity near $[\mathrm{Fe} / \mathrm{H}]=-1$ (on the metallicity scale of Zinn and West 1984 which is used throughout this paper). The clusters more metal poor than $[\mathrm{Fe} / \mathrm{H}]=-1$ are spread throughout the halo to distances approaching $20 \mathrm{kpc}$ from the galactic plane (the much more distant clusters, e.g., Pal 3 and 4, are discussed later), whereas the more metal rich ones are confined to within $3.2 \mathrm{kpc}$ of the plane. This concentration of the metal-rich clusters near the galactic plane is not simply a consequence of their concentration toward the galactic center, for there are several metal-rich clusters, e.g., M 71 and NGC 5927, that lie at substantial distances from the galactic center and yet close to the galactic plane. Using Frenk and White's (1982) method of analyzing the positions of the clusters on the sky, Zinn (1985) has shown that the metal-rich and metal-poor clusters near the galactic center as well as those at angular distances greater than 20 degrees from the center have different flattenings toward the galactic plane and that these differences are statistically significant (>958 confidence). This analysis suggests that the dividing line between the flattened and essentially unflattened groups occurs at $[\mathrm{Fe} / \mathrm{H}]=-0.8$, and hence, that roughly 258 of the cluster sample belongs to a disk system, while the remaining 758 belongs to the halo.

The kinematical properties of the clusters also change near $[\mathrm{Fe} / \mathrm{H}]=-0.8$ (Zinn 1985), as expected if the flattening of the metal-rich group is real. The clusters more metal poor than -0.8 are rotating slowly as a group around the galactic center $\left(V_{\text {rot }}=50 \pm 22\right.$ $\mathrm{km} / \mathrm{s}$ ) and have a large line-of-sight velocity dispersion ( $\sigma_{\text {los }}=113 \pm 9$ $\mathrm{km} / \mathrm{s})$. In contrast, the metal-rich clusters have the large rotation $\left(V_{\text {rot }}=153 \pm 29 \mathrm{~km} / \mathrm{s}\right)$ and small dispersion $\left(\sigma_{\text {los }}=71 \pm 11 \mathrm{~km} / \mathrm{s}\right)$ of a disk system. The more recent analysis by Hesser, Shawl, and Meyer (1986) of slightly different samples of clusters also shows that the metal-rich and metal-poor clusters have distinctly different kinematics. Cudworth (1984) has measured the proper motion of the metal-rich cluster $M 71$, and as expected from these analyses, its space velocity is typical of the old disk stellar population.

Additional evidence for two populations of clusters is provided by the distribution of the clusters over $[\mathrm{Fe} / \mathrm{H}]$. This distribution is bimodal (Harris and Canterna 1979), and the valley between its two peaks occurs near the dividing line in metallicity between the halo and 
disk subsystems (Freeman and Norris 1981; Zinn 1985).

There may be two populations of metal-poor clusters. Harris (1976) and Zinn (1985) have noted that the decline in the number density of globular clusters $(\phi)$ with increasing galactocentric distance ( $R$ ) is erratic beyond $R=20 \mathrm{kpc}$. From 3 to $20 \mathrm{kpc}, \phi$ decreases as $\mathrm{R}^{-3.5}$. It has a steeper decrease between 20 and $35 \mathrm{kpc}$, and between 35 and $60 \mathrm{kpc}$, there are no known clusters. There are, however, seven clusters in the range 60 to $110 \mathrm{kpc}(\mathrm{Pal} 3, \mathrm{Pal} \mathrm{4,} \mathrm{Pal}$ 14, Pal 15, AM-1, Eridanus, NGC 2419). This number is much larger than expected if $\phi$ declines smoothly beyond $20 \mathrm{kpc}$. With the exception of NGC 2419, this group consists of very low luminosity clusters that are hard to see on sky survey plates. It is conceivable, therefore, that additional clusters of this kind remain to be discovered, which would make the behavior of even more peculiar. One interpretation of these data is that the galactic halo ends at $R \approx 40 \mathrm{kpc}$ and the more distant clusters constitute a separate population of objects (Harris 1976). The recent work of Saha (1985) on the density of RR Lyrae variables in the halo appears to be consistent with this hypothesis, for he has found that their number density also falls off more rapidly between 20 and $33 \mathrm{kpc}$ (the limit of his survey) than at smaller distances. Obviously, it is important to extend as far as possible this survey and others of different samples of halo stars.

\section{METALLICITY GRADIENTS}

If the whole sample of globular clusters in the Galaxy is considered, then there is a steep decline in the mean value of $[\mathrm{Fe} / \mathrm{H}]$ with increasing $R$ because the metal-rich globular clusters are concentrated near the galactic center. But, as was pointed out above, there exist distinct halo and disk subsystems of clusters that undoubtedly evolved in different ways. If these systems are considered separately, then it is much less clear that gradients in metal abundance exist in either one.

\subsection{The Halo Gradient}

The question of the size of the gradient in the halo population of globular clusters has had a controversial history. Searle and Zinn (1978) concluded that there is no sign of a metallicity gradient in the halo beyond $\mathrm{R}=8 \mathrm{kpc}$. Harris and Canterna (1979) and more recently Pilachowski (1984) have contested this conclusion, for they found evidence for a gradient running from the galactic center out to 100 kpc. The large gradient found by these authors is a consequence of their samples containing the metal-rich disk clusters and the poor quality of the $[\mathrm{Fe} / \mathrm{H}]$ measurements then available for the clusters more distant than $R=60 \mathrm{kpc}$. This gradient nearly disappears once the disk clusters are removed from the sample of halo clusters and the most recent measurements of $[\mathrm{Fe} / \mathrm{H}]$ are adopted for the very distant clusters (see Zinn 1985 and 1986). 
There is nonetheless substantial evidence for a small gradient in the halo. The clusters in the zones $0<\mathrm{R}<7 \mathrm{kpc}$ and $7<\mathrm{R}<40 \mathrm{kpc}$ have significantly different distributions over [Fe/H] (Zinn 1985), even though in these zones the mean values of $[\mathrm{Fe} / \mathrm{H}]$ are not very different $(-1.50 \pm 0.05$ and $-1.69 \pm 0.08$ respectively). In the $7<R<$ $40 \mathrm{kpc}$ zone, there is no evidence for a gradient, although with a sample of only 36 clusters, which includes nearly all the known clusters in this zone, it is impossible to rule out a very small gradient. The clusters more distant than $60 \mathrm{kpc}$ have a distribution over $[\mathrm{Fe} / \mathrm{H}]$ that is essentially the same as the clusters in the $7<R<40$ kpc zone, which may be evidence that they do not constitute a separate population after all. At every value of $R$ in the halo population, there is a wide range of $[\mathrm{Fe} / \mathrm{H}]$ which has a standard deviation of approximately 0.3. This large dispersion and the small size of the gradient suggest that the collapse of the halo was chaotic and did not proceed with a smooth buildup of metals as its radius shrank.

The metallicity gradient exhibited by the RR Lyrae variables in galactic fields is in good agreement with the globular cluster observations. The observations of $R R$ Lyrae variables in the $7<R<40$ kpc zone (Butler, Kinman, and Kraft 1979; Butler, Kemper, Kraft and Suntzeff 1982; Saha 1985) have not revealed any evidence for a gradient. There is a slightly steeper gradient between the variables in the $0<R<7$ and $7<R<40 \mathrm{kpc}$ zones (see Kinman, Kraft, Friel and Suntzeff 1985) than between the globular clusters. Zinn (1986) has argued that this may not be evidence for a difference between the cluster and field populations, but a consequence of the influence that the second parameter effect has on the $[\mathrm{Fe} / \mathrm{H}]$ distribution of the $R R$ Lyrae variables.

\subsection{The Disk Gradient}

In his description of the disk system, Zinn (1985) presented some meager evidence for metallicity gradients with distance from the galactic plane and with R. More recently, Armandroff and Zinn (1986) have measured the infrared $\mathrm{Ca}$ II triplet in the spectra of many of the most metal-rich clusters. While the agreement with previous estimates of metallicity by other means is generally very good, the agreement with the values that Malkan (1982) obtained from infrared photometry is poor, and several of the clusters that he suggested were very metal rich turn out not to be so extreme. When these revisions are made to the data considered by Zinn (1985), the evidence for metallicity gradients disappears. This does not mean that gradients do not exist. The distance moduli of many of the metal-rich clusters have not been measured or are uncertain, and until these deficiencies are corrected, one cannot be certain that distance errors have not smeared out the gradients.

\section{HORIZONTAL-BRANCH MORPHOLOGY}

Theoretical calculations have shown that the morphology of the 
horizontal branch (HB) is sensitive to a large number of parameters, including cluster age, helium abundance, metallicity (primarily the abundances of $\mathrm{C}, \mathrm{N}$, and 0 ), and the rate of core rotation (affects the size of the He burning core and the amount of mass lost on the giant branch). If all of these parameters save metallicity were fixed or were monotonic functions of metallicity, then the HB's of globular clusters would be well behaved. When going from metal-poor to metal-rich clusters, one would see the bulk of the HB stars shift from the blue side of the instability strip to the red side. Instead one sees a large scatter in HB morphology at each metallicity, which indicates that something besides metallicity is varying from cluster to cluster. This additional parameter is called the second parameter, and it is a very real possibility that this effect is actually caused by more than one of the candidate second parameters mentioned above.

What links the second parameter effect to the evolution of the Galaxy is the fact that the size of the effect varies with galactocentric distance (see Zinn 1986 for a review). The effect is largest among the very distant clusters $(60<\mathrm{R}<110 \mathrm{kpc})$. Of the seven clusters in this zone, which are all metal poor $([\mathrm{Fe} / \mathrm{H}]<-1)$, the HB's of 5 are very red (i.e., they resemble that of 47 Tuc, a much more metal rich cluster) while the remaining 2 have the blue HB's expected of metal-poor clusters. The variation in $\mathrm{HB}$ morphology among the clusters in the $7<R<40 \mathrm{kpc}$ zone is smaller, but still appreciable at every metallicity. In the $0<\mathrm{R}<7 \mathrm{kpc}$ zone, there is little evidence that the second parameter effect exists at all, for $H B$ morphology varies smoothly with $[\mathrm{Fe} / \mathrm{H}]$.

The origin of this variation with $R$ has not been identified. Although Peterson's (1985) discovery of a correlation between the frequency of rotation among the $\mathrm{HB}$ stars in a cluster and its $\mathrm{HB}$ morphology suggests that core rotation is a second parameter, it is not clear why the amount of core rotation should vary from cluster to cluster in the outer halo and at the same time should be nearly uniform among the clusters of the same metal abundance in the inner halo. Core rotation is nonetheless a leading candidate for the origin of other peculiarities of HB morphology (see, for example, Buonanno, Corsi and Fusi Pecci 1985). Searle and Zinn (1978) have argued that differences in age can explain in a straight forward way the dependence of the second parameter effect on $R$. In their picture, the inner halo evolved rapidly, while the outer halo formed over $a \approx 4$ billion year period (see also Zinn 1980).

Unfortunately, the differences in age predicted on the basis of HB morphology are smaller than the precisions with which clusters can be dated. To illustrate this, let us consider the clusters NGC 288 and 362 , which have very nearly the same metallicities $([\mathrm{Fe} / \mathrm{H}]=-1.40$ and -1.27 , respectively, $\mathrm{Zinn}$ and West 1984) and yet have very different $\mathrm{HB}^{\prime} \mathrm{s}(\mathrm{B} /(\mathrm{B}+\mathrm{R})=1.00$ and 0.05 , respectively, Zinn 1980). Buonanno's (1986) compilation of measurements of the difference in bolometric magnitude between the $\mathrm{HB}$ and the main-sequence turn off, $\Delta \mathrm{M}_{\text {bol }}$ (TO-RR), 
lists values of 3.50 and 3.31 , respectively, for 288 and 362 . If one or another of these clusters has an age near 15 billion years, as commonly found for globular clusters (e.g., Buonanno 1986), then the difference in $\Delta \mathrm{M}_{\text {bol }}(\mathrm{TO}-\mathrm{RR})$ between them suggests that they differ in a age by about 2.4 billion years, in the sense that 288 is the older cluster (assuming they have the same abundance of $\mathrm{He}$ ). Theoretical calculations of synthetic HB's indicate that this difference in age can account for the observed difference in HB morphology (see fig. 1 in Rood and Seitzer 1982). However, this cannot be considered proof that the second parameter is age, for the uncertainty in each of the values of $\Delta \mathrm{M}_{\text {bol }}(\mathrm{TO}-\mathrm{RR}$ ) is \pm 0.15 (Buonanno 1986); hence, there is no reason to believe that their turn offs actually differ in luminosity. If measurements of the same precision could be obtained for the red $\mathrm{HB}$ clusters in the $60<\mathrm{R}<110 \mathrm{kpc}$ zone, then there is some hope that the age hypothesis could be tested. On the basis of their HB morphologies, these clusters are expected to be $\geq 4$ billion years younger than blue HB clusters of the same metallicity. Their turn offs are, however, too faint to be measured at present with the required precision.

\section{AGES AND THE AGE-METALLICITY RELATION}

Despite the progress produced by the advent of CCD detectors and the calculations of new theoretical isochrones, the dating of globular clusters has reached a plateau and very desirable quantities, such as the age-metallicity relation, remain beyond our grasp. To describe the current impasse, it is convenient to discuss the dating procedure that compares the luminosity of the main-sequence turn off with values given by theoretical isochrones. Because this method is insensitive to errors in the reddenings of the clusters and in the colors of the isochrones, it may be superior to the method of fitting the isochrones directly to the color-magnitude diagram (CMD). In any case, the following uncertainties afflict both methods.

Observationally, the luminosity of the main-sequence turn off is estimated in two steps. From a cluster's CMD, the quantity $\triangle M_{\text {( }}$ (TO-RR) is measured. Then a value for the absolute magnitude of the $R R$ Lyrae variables $\left(M_{v}(R R)\right)$, appropriate for the cluster's metal abundance, is adopted. The addition of these quantities yields, of course, the luminosity of the turn off, and this in turn yields an age from the theoretical calculations once a value of the abundance of $\mathrm{He}(\mathrm{Y})$ is adopted. The major uncertainty in the age that one obtains stems from the current debate regarding the dependence of $M_{(}(R R)$ on $[\mathrm{Fe} / \mathrm{H}]$. For example, with the assumption that $M_{(}(R R)$ is the same for all metallicities, one obtains a very large range in age (22.6 to 13.6 billion yrs, if $M_{v}(R R)=+0.6$ ) over the metallicity range of the halo, while with the assumption that $\Delta \mathrm{M}_{\mathrm{V}}(\mathrm{RR}) / \Delta[\mathrm{Fe} / \mathrm{H}]=0.35$ (Sandage 1982), one obtains no variation in age at all (see Zinn 1986). Both of these $\mathrm{M}_{\mathrm{v}}(\mathrm{RR})-[\mathrm{Fe} / \mathrm{H}]$ relations are supported by observations, and it is impossible at present to rule out either one. In addition, there is 
the possibility that $\Delta \mathrm{M}_{\mathrm{bol}}(\mathrm{TO}-\mathrm{RR})$ varies with $[\mathrm{Fe} / \mathrm{H}]$. While the present observations suggest that it does not (Buonanno 1986), a variation that yields a 3 billion year difference in age between $[\mathrm{Fe} / \mathrm{H}]=-2.5$ and -0.8 cannot be excluded. Furthermore, the adopted value of $\mathrm{Y}$ may be incorrect, and $\mathrm{Y}$ may vary with $[\mathrm{Fe} / \mathrm{H}]$. The isochrones may contain errors and may be for the wrong mixture of elements (e.g., wrong $\mathrm{CNO} / \mathrm{Fe})$. At present, therefore, the ages of globular clusters do not provide a precise chronology for the collapse of the Galaxy.

In spite of these problems, their ages do yield precious information. As many authors have remarked (e.g., Sandage 1982), the ages of globular clusters appear to be inconsistent with the age of the universe unless the value of the Hubble constant is no larger than about $50 \mathrm{~km} / \mathrm{sec} / \mathrm{Mpc}$. In addition, the ages that are obtained for the two clusters of the metal-rich disk system that can be dated at present, M71 and 47 Tuc, are identical to the ages obtained for some halo clusters, which suggests that there was not a large gap in time between the formation of the halo and the galactic disk, or at least the thick disk.

6. ARE THE GLOBULAR CLUSTERS GOOD TRACERS OF THE GALAXY'S STELLAR POPULATIONS?

This is not an idle question, because substantial evidence exists for differences between the globular cluster and stellar populations of giant elliptical galaxies (see the review by $W$. Harris 1987). If the properties of the globular clusters in the Galaxy are not shared by the field stars, then it would appear that under certain conditions the formation of globular clusters was favored over stars, as suggested, for example, by Fall and Rees (1985).

Recently, there have been a number of comparisons of the spatial distributions, kinematics, and metal abundance distributions of globular clusters and samples of stars, with the result that, at present, there appears to be no firm reason to reject the hypothesis that they are members of the same populations (see Hartwick 1983; Zinn 1985, 1986; Norris 1986; Freeman 1986 and references therein). The globular clusters that are more metal poor than $[\mathrm{Fe} / \mathrm{H}]=-0.8$ appear to be similar in these properties to halo stars. The more metal-rich clusters have the metallicities and kinematics of the Gilmore and Reid (1983) thick disk population (see Norris 1986; Freeman 1986). The scale height of the metal-rich clusters appears to be smaller than that inferred for the thick disk stars $(\approx 500$ pc as opposed to $\approx 1500$ pc, see Zinn 1985), but its measurement is uncertain.

Norris (1986) has recently analyzed the kinematics of a large sample of metal-poor stars (i.e., $[\mathrm{Fe} / \mathrm{H}] \geq-0.6$ ), and has found that the dividing line between the metal-poor halo and the more metal-rich thick disk occurs at $[\mathrm{Fe} / \mathrm{H}]=-1.2$. This value is significantly lower than the value indicated by the globular clusters $(-0.8)$, and it is 
important to see if this difference is real or is merely a consequence of the errors in the measurements of $[\mathrm{Fe} / \mathrm{H}]$, which are presumably larger in Norris's heterogeneous sample. To do this, I have compiled a sample of 148 RR Lyrae variables near the Sun that have well determined radial velocities and spectroscopically measured values of Preston's (1959) $\Delta S$ parameter (the literature is ripe with $\Delta S$ values inferred from photometry, and many of these disagree with more recent spectroscopic ones). The variables have been put into the following groups: $\Delta \mathrm{S} \leq 2,2<\Delta \mathrm{S} \leq 4,4<\Delta \mathrm{S} \leq 6,6<\Delta \mathrm{S} \leq 08$, and $\Delta \mathrm{S}<8$, and the rotational velocity and line-of-sight velocity dispersion of these groups have been calculated by the Frenk and White (1980) technique, assuming the Local Standard of Rest rotates at $220 \mathrm{~km} / \mathrm{s}$. To transform the $\Delta \mathrm{S}$ measurements to the $[\mathrm{Fe} / \mathrm{H}]$ scale of Zinn and West (1984), the equation: $[\mathrm{Fe} / \mathrm{H}]=-0.16 \Delta \mathrm{S}-0.41$ (Zinn 1986) was used, which differs slightly from the one used by Norris for the same purpose. According to this equation, the typical standard deviation of a $\Delta S$ measurement translates into a deviation of $\approx 0.2 \mathrm{dex}$ in $[\mathrm{Fe} / \mathrm{H}]$, which is comparable to the precision of the measurements for the globular clusters.

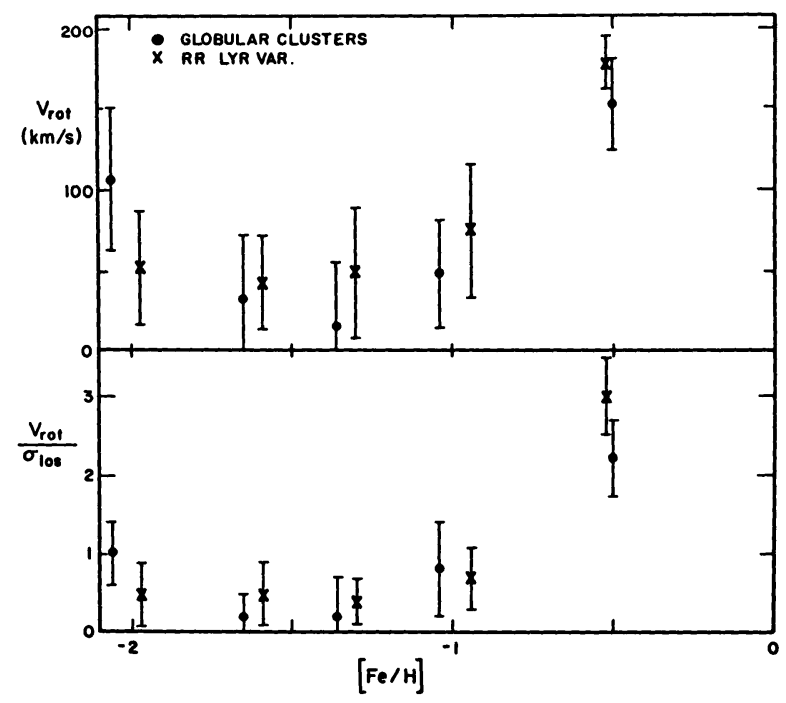

Fig. 1. For groups of globular clusters and RR Lyrae variables, the mean rotational velocity $\left(v_{\text {rot }}\right)$ and the ratio of $v_{\text {rot }}$ to the line-of-sight velocity dispersion $\left(\sigma_{\text {los }}\right)$ are plotted against the mean metallicity of the group.

Fig. 1 compares the calculations for the groups of RR Lyrae variables with ones for groups of globular clusters that have 
approximately the same mean metallicities (102 clusters in total, metallicities and radial velocities from zinn 1985). The quantity $\mathrm{V}_{\text {rot }} / \sigma_{\text {los }}$ is a diagnostic of rotational flattening, which is expected to be less than one in slowly rotating systems and greater than one in rotating disk systems. The data in Figure 1 indicate that the rotational properties of the clusters and the variables are very similar. In both samples, the change from halo to disk-like rotation appears to occur at $[\mathrm{Fe} / \mathrm{H}]<-1$, and $[\mathrm{Fe} / \mathrm{H}]=-0.8$ appears to be $a$ reasonable choice for the dividing line.

The data in Fig. 1 show that there is not a significant correlation between rotation and $[\mathrm{Fe} / \mathrm{H}]$ below $[\mathrm{Fe} / \mathrm{H}]=-0.8$. Norris (1986) found the same effect in his much larger sample of objects below $[\mathrm{Fe} / \mathrm{H}]=-1.2$. The lack of a correlation is what one expects on the basis of the observed absence of a steep metallicity gradient among the halo globular clusters and RR Lyrae variables, and it is consistent with Searle and Zinn's (1978) hypothesis that the halo formed out of the merger of several small systems. Sandage (1986), on the other hand, in his analysis of the metallicities and kinematics of a large sample of subdwarfs, has found a very significant correlation between rotation and $[\mathrm{Fe} / \mathrm{H}]$, which he interprets as strong evidence in favor of Eggen, Lynden-Bell, and Sandage's (1962) picture of progressive metal enrichment as the collapse of the halo proceeded. Until the origin of the discrepancy between these analyses is identified (see Freeman 1986 for one possibility), it is not clear which of these pictures of the formation of the halo best fits the data.

This research was supported by NSF grant AST-8304034.

\section{REFERENCES}

Armandroff, T. E. and Zinn, R. 1986 in preparation.

Baade, W. 1958 in Stellar Populations, D. J. K. O'Connell, ed., North Holland, Amsterdam, p. 303.

Buonanno, R. 1986 Mem. Soc. Astron. Italiana, in press.

Buonanno, R., Corsi, C. E. and Fusi Pecci, F. 1985 Astron. Astrophys. 145, 97.

Butler, D., Kemper, E., Kraft, R. P. and Suntzeff, N. B. 1982 Astron. J. 87, 353 .

Butler, D., Kinman, T. D. and Kraft, R. P. 1979 Astron. J. $84,993$.

Cudworth, K. M. 1984 Astron. J. 90, 65.

Eggen, O. J., Lynden-Be11, D. and Sandage, A. 1962 Astrophys. J. 136,748 .

Fal1, M., and Rees, M. 1985 Astrophys. J. 298, 18.

Freeman, K. C. 1986 in Stellar Populations, C. A. Norman, A. Renzini, and M. Tosi, eds., Cambridge University Press, in press.

Freeman, K. C. and Norris, J. 1981 Ann. Rev, Astron. Astrophys. $19,319$. 
Frenk, C. S. and White, S. D. M. 1980 Monthly Notices Roy. Astron. Soc. $193,295$.

Frenk, C. S. and White, S. D. M. 1982 Monthly Notices Roy. Astron. Soc. 198,173 .

Gilmore, G. and Reid, N. 1983 Monthly Notices Roy. Astron. Soc. $202,1025$.

Harris, W. E. 1976 Astron. J., 81, 1095.

Harris, W. E. 1987 in IAU Symposium No. 126. Globular Cluster Systems in Galaxies, J. E. Grindlay and A. G. D. Philip, eds., Reidel, Dordrecht, p. 237.

Harris, W. E. and Canterna, R. 1979 Astrophys. J., 231, L19.

Hartwick, F. D. A. 1983 Mem. Soc. Astron. Italiana, 54, 51.

Hesser, J. E., Shaw1, S. J. and Meyer, J. E. 1986 Pub1. Astron. Soc. Pacific 98, 403.

Kinman, T. D. 1959 Monthly Notices Roy. Astron. Soc. 119, 538.

Kinman, T. D., Kraft, R. P., Friel, E. and Suntzeff, N. B. 1985 Astron. J., 90, 95 .

Malkan, M. A. 1982 in IAU Colloq. No. 68. Astrophysical

Parameters for Globular Clusters, A. G. D. Philip and D. S.

Hayes, eds., L. Davis Press, Schenectady, p. 533.

Maya11, N. U. 1946 Astrophys. J. 104, 290.

Morgan, W. W. 1956 Publ. Astron. Soc. Pacific 68, 509.

Morgan, W. W. 1959 Astron. J. 64, 432.

Norris, J. 1986 Astrophys. J. Supp1. 61, 667.

Peterson, R. C. 1985 Astrophys. J. Letters 294, L35.

Pilachowski, C. A. 1984 Astrophys. J., 281, 614.

Preston, G. W. 1959 Astrophys. J., 130, 507.

Rood, R. T. and Seitzer, P. 0. 1982 in IAU Colloq. No. 68, Astrophysical parameters for Globular Clusters, A. G. D.

Philip and D. S. Hayes, eds., L. Davis Press, Schenectady, p. 369 .

Saha, A. 1985 Astrophys. J. 289, 310.

Sandage, A. 1982 Astrophys. J. 252, 553.

Sandage, A. 1986 in Stellar Populations, C. A. Norman, A.

Renzini and M. Tosi, eds., Cambridge University Press, in press.

Searle, L. and Zinn, R. 1978 Astrophys. J., 225, 357.

Shapley, H. 1918 Publ. Astron. Soc. Pacific 30, 42.

Zinn, R. 1980 Astrophys. J., 241, 602 .

Zinn, R. 1985 Astrophys. J., 293, 424.

Zinn, R. 1986 in Stellar Populations, C. A. Norman, A.

Renzini and M. Tosi, eds., Cambridge University Press, in press.

Zinn, R. and West, M. J. 1984 Astrophys. J. Supp1. 55, 45. 


\section{DISCUSSION}

COHEN: Although there are uncertainties of $2 \times 10^{9}$ years in globular cluster ages, if one assumes they all have same He abundance, the relative ages between clusters should be better. (If one is not willing to assume this, all cluster distances are suspect, anyway.) You count a mean age difference of at least $2 \times 10^{9}$ years between cluster at $7 \mathrm{kpc}$ and at $40 \mathrm{kpc}$ to explain the second parameter problem. Increasing numbers of $\mathrm{CM}$ diagrams for globular clusters in outer halo now exist and show the same age for globular clusters. Given better statistics, can one eliminate age as the second parameter?

ZINN: I will stick by my comment that the present measurements of the ages of globular clusters lack sufficient precision to test the hypothesis that cluster age is the dominant second parameter. The age range is predicted to be $\sim 2 \times 10^{9}$ for the clusters in the $7<\mathrm{R}<40$ kpc zone, which is comparable in size to the most optimistic claims made on the errors with which clusters can be dated. The ages of the very distant clusters with red horizontal branches are predicted to be as much as $4 \times 10^{9}$ yrs younger than blue horizontal-branch clusters. While, in principle, this difference should be measurable, it is not possible at present to construct CM diagrams of the required precision for the very distant clusters.

OSTRIKER: Could you say something further about the similarity or difference between the halo stars and the halo globular clusters? Specifically, is it possible, from chemical considerations, that most of the halo stars originated in dissolved globular clusters?

ZINN: The existing data suggest that the field stars and the globular clusters have similar metallicity distributions, kinematics, and spatial distributions, therefore, I believe that it is reasonable to hypothesize that the field stars are debris of disrupted clusters or that the field stars and the globular clusters originated in larger systems, the proto-dwarf galaxies that I mentioned, which dissolved into the galactic halo.

ZINNECKER: On your picture of accretion of proto-dwarf galaxies to form the galactic halo: isn't there additional support for this picture from the finding that there seem to be subsystems of clusters having distinctly difference space motions (prograde and retrograde)?

ZINN: I am not convinced that there is firm evidence for prograde and retrograde groups of clusters (See Zinn, R. 1985, Astrophys. J., 293, 424.). The problem with this, as I see it, is that for most clusters it is very difficult to tell the direction of rotation from radial velocity measurements alone. Obviously, if we had in addition proper motion data, which may be provided some day by observations from spacecraft, we could tell the direction of rotation, and then it would be very interesting to look for groups of clusters that have similar 
space motions.

KING: For the distant galactic globulars, we need not wait for HST, which will provide resolving power but will do relatively little for limiting magnitude. They should be done with ground-based observations, concentrating on good seeing. Our existing telescopes will collect enough photons; reduction time is more important than telescope time.

ZINN: To date the very distant clusters require photometry of $\sim 28$ precision for $V \sim 25-26$. This is very difficult, in my opinion, from the ground because it requires truly exceptional seeing. Christian and Heasley (1986, Astrophys. J.) have produced, from data obtained in good seeing, a very pretty CM diagram for the distant globular cluster $\mathrm{Pal}$ 4. However, this diagram does not place strong constraints on the age of $\mathrm{Pal}$ 4. I'm not sure one can do much better than this at the present time.

CARNEY: To partially answer Jerry's question and previewing my talk later, the field and cluster stars seem to show about the same overall metallicity distribution and detailed elemental abundance patterns. At least, there is no strong evidence they differ significantly. 